\title{
Long noncoding RNA UCA1 from hypoxia- conditioned hMSC-derived exosomes: a novel molecular target for cardioprotection through miR-873-5p/XIAP axis
}

Ling Sun ${ }^{1,2}$, Wenwu Zhu', Pengcheng Zhao', Qingjie Wang², Baohan Fan', Yegian Zhu', Yao Lu', Qiushi Chen', Jian Zhang ${ }^{1}$ and Fengxiang Zhang (D)

\begin{abstract}
Exosomes (Exo) secreted from mesenchymal stem cells (hMSCs) are protective against myocardial injury. The purpose of the study was to investigate the role and mechanisms by which exosomes promote cardiomyocyte survival and function following myocardial infarction (MI). hMSCs were cultured under hypoxic and normoxic conditions. Hypoxiaconditioned hMSC-derived exosomes (Hypo-Exo) and normoxic-conditioned hMSC-derived exosomes (Nor-Exo) were collected and intramyocardially injected into rats with Ml. The therapeutic effects of Hypo-Exo and Nor-Exo were evaluated after 4 weeks. Quantitative real-time PCR (qRT-PCR) was used to detect the expression of candidate long noncoding RNA urothelial carcinoma associated 1 (IncRNA-UCA1) in Nor-Exo and Hypo-Exo. Intramyocardial injection of IncRNA-UCA1-knockdown-Hypo-Exo in a rat model of MI was then performed and the cardiac function was characterized. The target and downstream of the molecular mechanism IncRNA-UCA1 was disclosed by luciferase reporter assays and western blot. Circulating exosomal InCRNA-UCA1 level in AMI patients and healthy volunteers was assessed. We found that (1) hMSC exosomal (from hypoxic and normoxic conditions) cardioprotection in vitro and in vivo correlated with the presence of encapsulated IncRNA-UCA1 in exosomes; (2) IncRNA-UCA1 targeted miR-873 via sponging, reducing the latter's suppressive effects on its target XIAP, and this translated into AMPK phosphorylation and increased level of the antiapoptotic protein $\mathrm{BCL}$; and (3) plasma derived from patients with AMI contained exosomes enriched with the IncRNA-UCA1, unlike that from normal subjects. This study demonstrates that Hypo-Exo IncRNA-UCA1 plays a cardioprotective role via the miR-873-5p/XIAP axis and circulating exosomal IncRNA-UCA1 may be a promising novel biomarker for the diagnosis of AMI.
\end{abstract}

\section{Introduction}

Ischemic heart disease is a leading cause of death ${ }^{1}$ of which acute myocardial infarction (AMI) is an important cause, leading to a poor prognosis ${ }^{2,3}$. Coronary artery occlusion leads to hypoxia with consequent death of

\footnotetext{
Correspondence: Fengxiang Zhang (njzfx6@njmu.edu.cn)

'Section of Pacing and Electrophysiology, Division of Cardiology, The First

Affiliated Hospital with Nanjing Medical University, Nanjing, China

${ }^{2}$ Department of Cardiology, The Affiliated Changzhou No. 2 People's Hospital

of Nanjing Medical University, Changzhou, China

The authors contributed equally: Ling Sun, Wenwu Zhu, Pengcheng Zhao

Edited by Y. Shi
}

cardiomyocyte ${ }^{4}$. Currently, stent insertion and thrombolysis are the main treatment methods ${ }^{5,6}$. Nonetheless, they can be associated with reperfusion injury, including myocardial cell death and microvascular injury ${ }^{7}$. An effective alternative therapy for myocardial infarction (MI) that can reduce cardiomyocyte loss is therefore urgently required.

Transplantation of stem cells has been reported to promote cardiac function after $\mathrm{MI}^{8}$. Recently, several studies have confirmed this cardioprotective function of stem cells, largely achieved by secretion of paracrine

\section{(c) The Author(s) 2020}

(c) (i) Open Access This article is licensed under a Creative Commons Attribution 4.0 International License, which permits use, sharing, adaptation, distribution and reproduction c. in any medium or format, as long as you give appropriate credit to the original author(s) and the source, provide a link to the Creative Commons license, and indicate if changes were made. The images or other third party material in this article are included in the article's Creative Commons license, unless indicated otherwise in a credit line to the material. If material is not included in the article's Creative Commons license and your intended use is not permitted by statutory regulation or exceeds the permitted use, you will need to obtain permission directly from the copyright holder. To view a copy of this license, visit http://creativecommons.org/licenses/by/4.0/. 
factors including exosomes $(\text { Exo })^{9}$. Exo are small $(30-200 \mathrm{~nm})$ endogenous membrane vesicles secreted by most cells ${ }^{10}$ and transmit a variety of signaling molecules including proteins, microRNAs (miRNAs), and long noncoding RNAs (lncRNAs) ${ }^{11}$ as well as mediating cellto-cell communication and crosstalk between organs. In addition, compared with stem cell transplantation, Exo have the advantages of a low tumorigenic potential, minimal immunogenicity, and do not lead to teratoma formation. They are therefore considered to be a promising cell-free therapy for cardiac repair ${ }^{10}$.

A recent study suggested that mesenchymal stromal cell-derived Exo could attenuate injury through miR-182regulated macrophage polarization ${ }^{12}$. Another study demonstrated that injection of hypoxic-conditioned human mesenchymal stem cell (hMSC)-derived Exo (Hypo-Exo) in MI mice improved the therapeutic effects of normoxia-conditioned hMSC-derived Exo (Nor-Exo) via miR-125b-5 $p^{12,13}$. Nonetheless few studies have reported the therapeutic effects of MSC-derived Exo in AMI via mediating $\operatorname{lncRNA}{ }^{14}$. In the current study, we investigated the protective role of Hypo-Exo using a rat model of MI. We established that Hypo-Exo is enriched with IncRNA urothelial carcinoma associated 1 (lncRNAUCA1) that exhibits an antiapoptotic effect in vivo and in vitro. We revealed that knockdown of lncRNA-UCA1 in Hypo-Exo significantly increased the infarct area and promoted cardiomyocyte apoptosis. In addition, circulating exosomal lncRNA-UCA1 level in AMI patients has the potential to serve as a diagnostic biomarker.

\section{Materials and methods}

\section{Ethical considerations}

The present study was conducted in accordance with the principles of the Declaration of Helsinki and approved by the Ethics Committee of the First Affiliated Hospital and Changzhou No. 2 People's Hospital of Nanjing Medical University.

\section{Patients}

Blood samples were obtained from 26 patients with confirmed AMI (from the cardiac care unit) and 26 healthy volunteers (from the health examination center) at Changzhou No. 2 People's Hospital of Nanjing Medical University. Informed consent was obtained from all study subjects.

\section{Cell culture, hypoxia exposure, and transfection}

All cells were incubated at $37^{\circ} \mathrm{C}$ in $5 \% \mathrm{CO}_{2}$ and $21 \% \mathrm{O}_{2}$ under normal condition. hMSCs from healthy adults were purchased from Cambrex BioScience and cultured in $\alpha$ minimal essential medium ( $\alpha$-MEM) with $10 \%$ fetal bovine serum (FBS). The surface marker of hMSCs was determined using flow cytometry. In the hypoxic experiments, hMSCs were transferred into a hypoxic chamber (Thermo Forma, USA) with $1 \% \mathrm{O}_{2}, 5 \% \mathrm{CO}_{2}$, and 94\% $\mathrm{N}_{2}$ for 48 and $12 \mathrm{~h}$, and Exo were isolated. H9c2 cardiomyoblasts (American Type Culture Collection) were cultured in Dulbecco's modified Eagle's medium (DMEM) (Gibco, USA) with 10\% FBS. In the hypoxic and serum deprivation $(\mathrm{H} / \mathrm{SD})$ experiments, H9c2 cells were cultured with DMEM with no glucose (11966025, Gibco, USA) in $94 \% \mathrm{~N}_{2}, 5 \% \mathrm{CO}_{2}$, and $1 \% \mathrm{O}_{2}$ for $12 \mathrm{~h}$.

Transfection of lncRNA silencer $(200 \mathrm{nmol} / \mathrm{l})$, miRNA mimics $(100 \mathrm{nmol} / \mathrm{l})$, miRNA inhibitor $(200 \mathrm{nmol} / \mathrm{l})$, and their negative controls $(100-200 \mathrm{nmol} / \mathrm{l})$ was carried out using riboFECT ${ }^{\mathrm{TM}}$ CP Reagent (Ribobio, China) according to the manufacturer's instructions.

\section{Exo isolation and characterization}

Human blood samples were collected in an ethylene diamine tetraacetic acid anticoagulant tube and centrifuged immediately $(3000 \times g, 15 \mathrm{~min})$. Supernatant (plasma) was obtained and thawed on ice. Ribo ${ }^{\mathrm{TM}}$ Exosome Isolation Reagent (a precipitation reagent for plasma or serum, C10110-2, Ribobio, China) was used for Exo isolation according to the manufacturer's instructions. In brief, plasma samples were centrifuged at $2000 \times g$ for $20 \mathrm{~min}$ to remove cells and cell debris. Clarified plasma samples $(3 \mathrm{ml})$ were then transferred to a clean tube containing $1 \mathrm{ml}$ Ribo Exosome Isolation Reagent. The mixtures were incubated overnight at $4{ }^{\circ} \mathrm{C}$ and then centrifuged at $15,000 \times g$ for $2 \mathrm{~min}$. Supernatant was aspirated and the Exo resuspended in $100 \mu \mathrm{l} 1 \times$ phosphate-buffered saline (PBS).

hMSCs $\left(1 \times 10^{6}\right)$ were grown in a cell culture flask (T25, Corning, USA) to $80 \%$ confluence. Cells were then washed once with PBS and treated with $5 \mathrm{ml}$ Exo-free FBS (Gibco) for 2 days. Cell culture medium $(5 \mathrm{ml})$ was harvested in a $15-\mathrm{ml}$ conical tube and centrifuged at $1500 \times g$ for $30 \mathrm{~min}$ to remove cells and debris. Cell-free media was transferred into another $15 \mathrm{ml}$ conical tube containing $2 \mathrm{ml}$ Ribo $^{\mathrm{TM}}$ Exosome Isolation Reagent (for cell culture media, C10130-2, Ribobio, China). Similarly, these mixtures were incubated overnight at $4{ }^{\circ} \mathrm{C}$ and then centrifuged at $2000 \times g$ for $30 \mathrm{~min}$. The supernatant was aspirated and the Exo resuspended in $100 \mu \mathrm{l}$ PBS.

A BCA Protein Assay Kit (Thermo Fishier Scientific, USA) was used to measure exosomal proteins. The surface markers of Exo were detected using western blotting with anti-TSG101, CD63, and CD81 antibodies (Abcam, UK). These Exo were fixed with $1 \%$ glutaraldehyde, applied on a carbon-coated copper grid, and then stained with $1 \%$ phosphotungstic acid. The samples were tested using a JEM-2100 transmission electron microscope (TEM; JEOL, Japan). Size distribution and concentration of particles was evaluated by nanoparticle tracking analysis (NTA) using ZetaView PMX 110 (Particle Metrix, 
Germany $)^{15}$. Brownian motion of particles was tracked and recorded by laser scattering microscopy. The diameters of the particles and size distribution data were analyzed using the Stokes-Einstein equation.

\section{Exo uptake assay}

To determine whether Exo could be absorbed by H9c2 cells, Exo were labeled with a red fluorescent dye (Dil, Invitrogen). Briefly, $1 \mu \mathrm{M}$ Dil was incubated with $100 \mu \mathrm{g}$ Exo for $15 \mathrm{~min}$. To remove excess dye, $3 \mathrm{ml} \mathrm{PBS}$ and Exosome Isolation Reagent were added, and the mixture was centrifuged at $1500 \times g$ for $30 \mathrm{~min}$. Supernatant was then aspirated, and the Dil-labeled Exo were resuspended in $100 \mu \mathrm{l} \mathrm{PBS}$. The Dil-labeled Exo were co-cultured with $\mathrm{H} 9 \mathrm{c} 2$ cells at $37^{\circ} \mathrm{C}$. The cells were subsequently washed with PBS and fixed with $4 \%$ paraformaldehyde for $15 \mathrm{~min}$. Fixed cells were washed with PBS and nuclei stained with 4',6-diamidino-2-phenylindole (DAPI, $0.5 \mu \mathrm{g} / \mathrm{ml}$; Invitrogen). A confocal microscope (Carl Zeiss, Germany) was used to observe the red signals in cells.

\section{Cell viability assay}

Cell viability was measured using the Cell Counting Kit8 (CCK-8, Dojindo, Japan). Briefly, H9c2 cells (2000 cells/ well) were seeded in 96-well plates and cultured with PBS or Exo $(200 \mu \mathrm{g} / \mathrm{ml})$ for $10 \mathrm{~h}$. Ten microliters of CCK-8 solution was added to each well. After $2 \mathrm{~h}$, absorbance was measured at $450 \mathrm{~nm}$ using a Synergy 2 microplate reader (Bioteck, USA). The percentage of viable cells was calculated with the control cells set as $100 \%$.

\section{Apoptosis assays}

Flow cytometry (KeyGEN Biotech, China) was used to assess cell apoptosis. H9c2 cells were cultured overnight at a seeding density of $1 \times 10^{5} / 6$-well tissue culture plates and treated with Exo or PBS before being subjected to hypoxia. To quantify the apoptotic cells, cells were washed with PBS and stained using an Annexin VFluorescein Isothiocyanate and Propidium Iodide Apoptosis Kit (KeyGen Biotech, China). The apoptotic cells were analyzed using the Flowjo Software version 10.0 (Tree Star, USA). TdT-mediated dUTP Nick-End Labeling (TUNEL) Apoptosis Detection Kit (Roche, USA) was also used to determine cell or tissue apoptosis according to the manufacturer's instructions. The percentage of apoptotic nuclei was calculated by dividing the total number of TUNEL-stained nuclei by the total number of TUNEL-positive nuclei.

\section{Quantitative real-time PCR (qRT-PCR)}

qRT-PCR was performed using the 7900HT Real-Time PCR Detection System (Thermo Fisher Scientific, USA). Reactions were performed in triplicate with each sample. Briefly, the total cellular and exosomal RNA was extracted using Trizol reagent (Life Technologies, USA). For quantification of IncRNA-UCA1 or X-linked inhibitor of apoptosis protein (XIAP) mRNA, first-strand cDNA was synthesized with random primers using a PrimeScript ${ }^{\mathrm{TM}} \mathrm{RT}$ Reagent Kit with gDNA Eraser (Takara, Japan). Stem-loop qRT-PCR was performed using a FastStart Essential DNA Green Master (Roche, USA). The cellular expression of lncRNA-UCA1 and XIAP mRNA was normalized to that of glyceraldehyde 3-phosphate dehydrogenase $(\mathrm{GAPDH})^{16}$. The exosomal level of lncRNAUCA1 was normalized to that of cel-miR-39 (C39) ${ }^{17,18}$. For quantification of miR-873-5p, cDNA was synthesized with an miRNA First-Strand cDNA Synthesis Kit (by stem-loop) (Vazyme Biotech, China). AceQ qPCR SYBR Green Master Mix (Vazyme Biotech, China) was then used for real-time PCR. The cellular miR-873-5p expression was normalized to U6. The relative expression was calculated using the following equation: relative gene expression $=2^{-(\Delta \text { Ctsample }-\Delta \text { Ctcontrol })}$. The primers are listed in Supplementary Table 1.

\section{Western blot}

Cells were lysed on ice for $30 \mathrm{~min}$ with lysis buffer. Total cell protein concentration was determined using a BCA Protein Assay Kit. The total protein $(30 \mu \mathrm{g})$ was resolved using sodium dodecyl sulfate-polyacrylamide gel electrophoresis (Invitrogen) and transferred to a polyvinylidene fluoride membrane (Roche). Membranes were blocked with $5 \%$ bovine serum albumin in TBS-Tween $(0.1 \%)$ and incubated against the desired antibodies. The primary antibodies BAX (5023, Cell Signaling Technology, USA), BCL-2 (ab196495, abcam, USA), p53 (21083, Signalway Antibody, USA), AMP-activated protein kinase (AMPK, 5831, Cell Signaling Technology), phosphorylation of AMPK (p-AMPK, 500815, Cell Signaling Technology), XIAP (14334, Cell Signaling Technology), GAPDH (5174, Cell Signaling Technology), TSG101 (14497, Proteintech, United States), CD63 (25682, Proteintech), CD81 (66866, Proteintech), and horseradish peroxidase-conjugated secondary antibody (Santa Cruz) were used. Bands were visualized using enhanced chemiluminescence reagents and analyzed using a gel documentation system (Bio-Rad Gel Doc1000 and Multi-Analyst version 1.1).

\section{Exosomal IncRNA-UCA1 knockdown}

For depletion of IncRNA-UCA1, hMSCs were stably transfected with GV248/UCA1 short hairpin RNA (shRNA) or GV248/control shRNA plasmid (GeneChem, China). The three-pooled shRNA sequences that yielded the greatest knockdown are listed in Supplementary Table 2. Seventy-two hours later, hMSCs were cultured in $\alpha$-MEM under hypoxic conditions for $48 \mathrm{~h}$. The Exo derived from hMSCs were then isolated, as described previously. 


\section{Luciferase assay}

The fragment of IncRNA-UCA1 $3^{\prime}$ untranslated region (UTR) cDNA containing the miR-873-5p-binding site was amplified by PCR. The amplified products were cloned in PGL3 vector (Promega, USA) immediately downstream of the stop codon of the luciferase gene. The mutant of lncRNA-UCA1 was constructed using the Takara MutanBEST Kit (Takara, Japan). For the luciferase assay, HEK-293T cells in 24-well plates were transfected with $200 \mathrm{ng} /$ well luciferase reporter constructs, $400 \mathrm{ng} /$ well miR-873-5p mimic, or mimic control using Lipofectamine 2000. SV-Renilla luciferase plasmids ( $5 \mathrm{ng} /$ well) served as the internal control. Cells were harvested at $24 \mathrm{~h}$ after transfection, and the luciferase activity detected using the Dual Luciferase Reporter Assay Kit (Promega, USA) as per the manufacturer's instructions. Firefly luciferase activities were normalized to Renilla luciferase activity.

\section{Animal experiments}

Male Sprague-Dawley rats ( 8 weeks of age, $n=6$ for each group) were obtained from the experimental animal center of Nanjing Medical University. All surgical procedures and animal care protocols were performed in accordance with the Guide for the Care and Use of Laboratory Animals published by the U.S. National Institutes of Health. Animal protocols were performed with approval of the Experimental Animals Ethics Committee of the First Affiliated Hospital of Nanjing Medical University (No. IACUC-1905024). The animals were randomly divided into different treatment groups. All surgeries and subsequent analyses were blinded for intervention.

\section{MI protocol, Exo delivery, and myocardial injury assessment}

All rats were anesthetized via intraperitoneal injection of $100 \mathrm{mg} / \mathrm{kg}$ ketamine combined with $10 \mathrm{mg} / \mathrm{kg}$ xylazine and then intubated and ventilated. The left anterior descending (LAD) coronary was ligated to induce $\mathrm{MI}^{14}$. One hundred microliters of Exo $(1 \mu \mathrm{g} / \mu \mathrm{l})$ or $100 \mu \mathrm{l}$ PBS were injected into the border zone of the infarcted heart at three locations. To evaluate cardiac function after treatment, transthoracic two-dimensional (2D) M-mode echocardiography was performed using a Vevo 2000 high-resolution micro-imaging system (Visual Sonic) 2 and 4 weeks after MI. The parameters of heart function were recorded in 2D and M-mode from the parasternal long-axis view at the papillary muscle level. Left ventricular end-diastolic dimension (LVEDD) and left ventricular end-systolic dimension (LVESD) were measured in at least three consecutive cardiac cycles. Left ventricular ejection fraction (LVEF) and left ventricular fractional shortening (LVFS) were used to evaluate the systolic function of the hearts. LVEF was calculated as
$\left[\left(\mathrm{LVEDD}^{3}-(\mathrm{LVESD})^{3}\right] /(\mathrm{LVEDD})^{3}\right] \times 100 \%$ and $\mathrm{LVFS}$ was calculated as [(LVEDD - LVESD)/LVEDD] $\times 100 \%$. All measurements were made by an independent blinded sonographer. Finally, rats were sacrificed by intraperitoneal administration of pentobarbital.

To evaluate whether Exo could be absorbed by the myocardium, Dil-labeled Exo were injected in rats with MI. Six hours later, rats were sacrificed. The hearts were dehydrated and frozen, and the ventricular papillary muscle was sliced into $6-\mu \mathrm{m}$-thick cryosections. The heart sections were immunofluorescent stained with $\alpha$-actin and then dyed with DAPI. Internalization of Exo into myocardium was observed by fluorescence microscope.

Infarct size was measured by Masson staining; myocardial apoptosis was determined by TUNEL staining using an In Situ Cell Death Detection Kit (Roche, USA).

\section{Statistical analysis}

Continuous and categorical variables are described as mean \pm SEM and percentages (\%), respectively. For continuous variables, Student's $t$ test (normal distribution data) or Mann-Whitney $U$ test (abnormal distribution data) were used. One-way analysis of variance (three groups) followed by Bonferroni's correction, if needed, were performed. For categorical variables, chi-squared test was used. Receiver operating characteristic (ROC) curve analysis was performed to discriminate AMI patients from healthy controls. The area under the ROC curve (AUC) was measured. All statistical tests were performed with the GraphPad Prism software version 5.0, and $P<0.05$ (two-sided) was considered statistically significant.

\section{Results}

Identification of Exo derived from hMSCs cultured under normoxic and hypoxic conditions

hMSCs were cultured under normoxic or hypoxic conditions. Supernatants were collected and Exo were extracted (Fig. 1a, c). Flow cytometry revealed that hMSCs were positive for CD44, CD73, and CD105 and negative for CD31, CD34, and CD45 (Fig. 1b). As shown in Fig. 1d, Exo showed typical lipid bilayer membraneencapsulated nanoparticles. Figure 1e demonstrates that the proteins of TSG101, CD81, and CD63 were positively expressed in normoxic and hypoxic Exo. NTA showed that the peak diameter of normoxic and hypoxic Exo was 96.7 and $97.9 \mathrm{~nm}$, respectively (Fig. 1f). The protein concentration of Hypo-Exo was increased significantly compared with that of Nor-Exo. In addition, NTA analysis revealed a similar trend, indicating an increase in the number of hMSC-derived Exo under hypoxic conditions. There were no significant differences in average size between Hypo-Exo and Nor-Exo (Supplementary Table 3). 
A

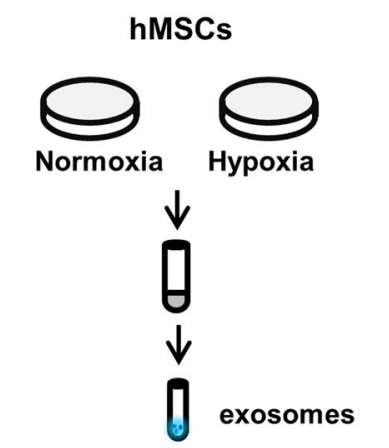

C

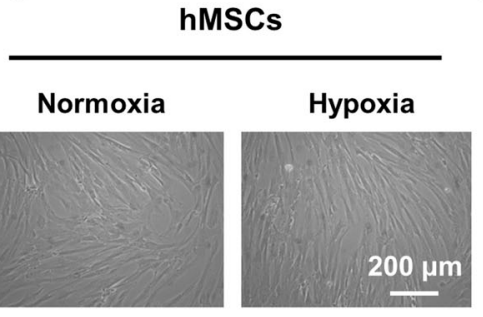

$\mathbf{F}$

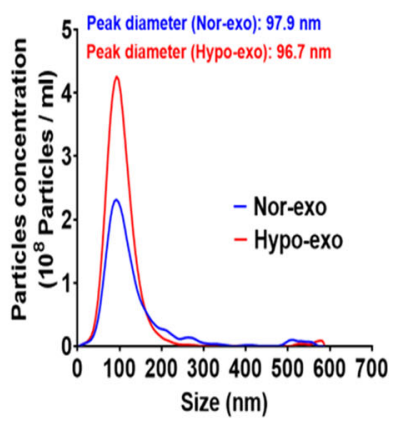

B
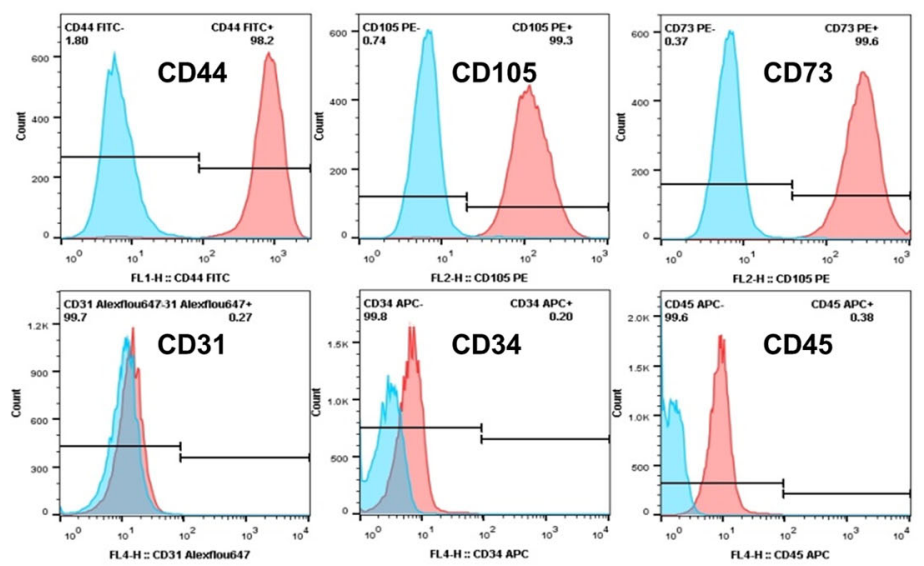

D

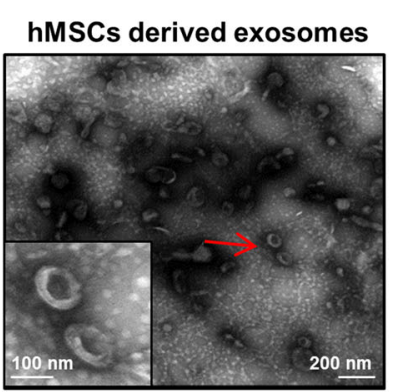

E

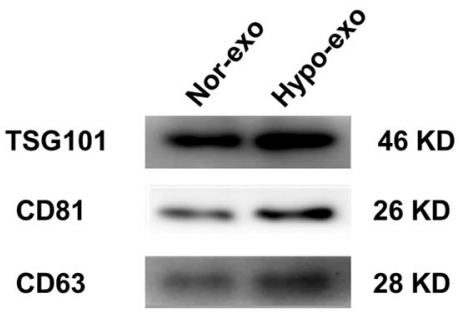

MERGE
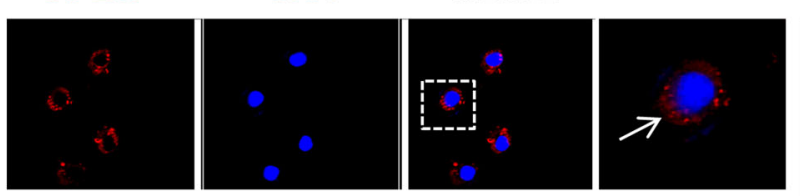

$24 \mathrm{~h}$
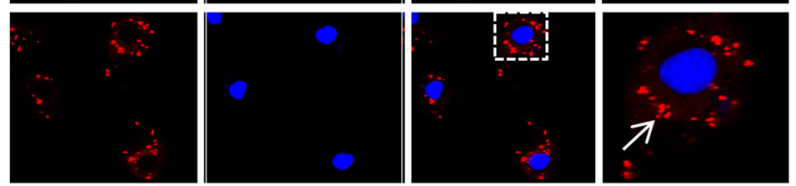

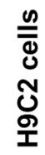

Control
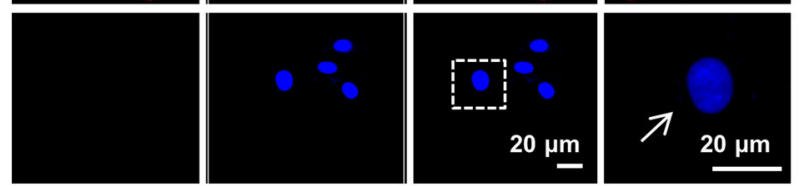

Fig. 1 Characterization of exosomes derived from hMSCs cultured under normoxic and hypoxic conditions. a Exosomes were extracted from mesenchymal stem cell (hMSC) supernatants under normal and hypoxic conditions. b Surface markers of hMSCs detected by flow cytometry: positive for CD44, CD105, and CD73; negative for CD31, CD34, and CD45. c Representative image showing the morphology of normoxic hypoxic hMSCs under light microscopy. d Electron micrograph-analyzed hMSC-derived exosomes. The red arrow indicates exosomes. Scale bar: $100 \mathrm{~nm}$. e Western blot showing the protein level of TSG101, CD63, and CD81 in normoxia-conditioned hMSC-derived Exo (Nor-Exo) and hypoxia-conditioned hMSCderived Exo (Hypo-Exo), respectively. $\mathbf{f}$ Size distribution of Nor-Exo and Hypo-Exo determined by nanoparticle tracking analysis (NTA). $X$ axis represents size of exosomes. $Y$ axis represents the particle concentration in $1 \mathrm{ml} \mathrm{PBS} \mathrm{(before} \mathrm{dilution).} \mathbf{g} \mathrm{H} 9 \mathrm{c} 2$ cardiomyocytes were cultured in the presence or absence (control) of Dil-labeled exosomes (red) at $37^{\circ} \mathrm{C}$ for 6 and $24 \mathrm{~h}$. The nucleus was stained with DAPI (blue). The white arrow indicates Dil-labeled exosomes absorbed by exosomes.

The Exo were labeled with Dil dye and co-cultured with H9c2 cells for 6 and 24h. Confocal microscopy showed that Dil-labeled Exo could be observed around the nucleus and appeared in a time-dependent manner
(Fig. 1g). Collectively, these results indicate that normoxic and hypoxic hMSCs could secrete Exo with common exosomal features and these Exo could be absorbed by H9c2 cells. 


\section{Hypo-Exo exerted better cardioprotection against} myocardial injury than Nor-Exo in vitro and in vivo

Nor-Exo, Hypo-Exo, and PBS were co-cultured with H9c2 cells under normal and H/SD conditions. Cell viability was significantly increased in the Nor-Exo and Hypo-Exo groups compared with the PBS group. Moreover, Hypo-Exo had an even better performance than Nor-Exo (Fig. 2a). Hypo-Exo and Nor-Exo also suppressed apoptosis when co-cultured with H9c2 cells under H/SD conditions (Fig. 2b and Supplementary Fig. 1). A rat model of AMI, achieved by permanent LAD artery ligation, was used to determine the cardioprotective effects of hMSC-derived Exo in vivo. Hypo-Exo, Nor-Exo $(100 \mu \mathrm{l}, 1 \mu \mathrm{g} / \mu \mathrm{l})$, or $100 \mu \mathrm{l}$ PBS were intramyocardially injected into rats at the time of MI (Fig. 2c). To observe whether Exo could be absorbed by cardiomyocytes, Dillabeled Exo was intramyocardially injected. Immunofluorescent staining for cardiomyocyte-specific ( $\alpha$-actin) antigens was performed. Dil-labeled Exo were colocalized with cardiomyocytes $6 \mathrm{~h}$ after injection, suggesting an efficient in vivo uptake of the Exo by cardiomyocytes (Fig. 2d). LVEF and LVFS were significantly improved in both Nor-Exo- and Hypo-Exo-injected animals compared with those injected with PBS. LVEF and LVFS were higher in the Hypo-Exo group than those in the Nor-Exo group (Fig. 2e). Quantification of the infarcted area $4 \mathrm{~h}$ post-MI indicated that Hypo-Exoinjected rats had a smaller fibrotic area than PBS-injected rats $(8.27 \% \pm 1.78 \%$ vs. $26.53 \% \pm 2.75 \%, P<0.001)$ and Nor-Exo-injected rats $(14.66 \% \pm 2.57 \%, P<0.05)$ (Fig. 2 f). These in vitro and in vivo data suggest that Hypo-Exo exerts better cardioprotective effects against myocardial injury than Nor-Exo.

\section{LncRNA-UCA1 was a key component of Hypo-Exo-induced cardioprotection}

To clarify whether IncRNA-UCA1 in Exo was induced by hypoxia, intracellular and exosomal lncRNA-UCA1 levels was detected using qRT-PCR. Hypoxia induced upregulation of lncRNA-UCA1 not only in hMSCs but also in hMSC-derived Exo (Fig. 3a, b). The level of IncRNA-UCA1 significantly decreased in hypoxic Exo treated with both RNase A and Triton X-100, not just RNase A, suggesting that IncRNA-UCA1 exists in Exo (Fig. 3c). H9c2 cells were then co-cultured with Hypo-Exo under $\mathrm{H} / \mathrm{SD}$ conditions. UCA1 silencer (si-UCA1 group) and its negative control (si-UCA1 NC group) were transfected into H9c2 cells. Inhibition of lncRNA-UCA1 attenuated the protective effects of Hypo-Exo in vitro (Fig. $3 \mathrm{~d}-\mathrm{g}$ ). We then detected apoptosis-related proteins and found that protein expression of P53, BAX, and cleaved-caspase- 3 significantly increased and the protein level of BCL-2 decreased remarkably in the siUCA1 group (Fig. 3h).
To gain mechanistic insight into the role of exosomal lncRNA-UCA1 in Hypo-Exo-induced cardioprotection in the rat MI model, lncRNA-UCA1 loss-of-function studies were performed in Hypo-hMSCs (Fig. 4a). Here an shRNA vector (shUCA1-Exo group) or shRNA negative control (shUCA1-NC-Exo group) was transfected into Hypo-hMSCs; green fluorescence was observed (Fig. 4b). Then Exo were isolated. In addition, qPCR analysis confirmed that shUCA1-Exo significantly reduced cellular and exosomal IncRNA-UCA1 level compared with shUCA1-NC-Exo (Fig. 4c), indicating successful transfection. LVEF and LVFS were significantly decreased in shUCA1-Exo-injected rats compared with shUCA1-NCExo-injected rats at the 14th and 28th day after MI (Fig. 4d). Figure 4e shows significantly increased apoptotic cells at the border zone in shUCA1-Exo-treated rats compared with shUCA1-NC-Exo-treated rats $(41.95 \% \pm$ $16.60 \%$ vs. $18.82 \% \pm 8.66 \%, P<0.001)$.

Quantification of fibrosis size of the hearts 4 weeks post-MI showed that shUCA1-NC-Exo-injected rats had a reduced size compared with shUCA1-Exo- and PBSinjected rats (Fig. 5a, b). As shown in Fig. 5c, shUCA1-Exo injection also increased the expression of $\alpha$-smooth muscle actin and Collagen I compared with shUCA1-NCExo injection after MI. The number of cleaved-caspase- $3^{+}$ and $\mathrm{BAX}^{+}$cells was also significantly lower in the shUCA1-NC-Exo group than in the shUCA1-Exo group (Fig. $5 \mathrm{~d}-\mathrm{g}$ ). Similar trends were revealed by western blot analysis (Fig. 5h). These results show that lncRNA-UCA1 is a key component of the cardioprotection afforded by Hypo-Exo in vitro and in vivo.

\section{LncRNA-UCA1 exerted cardioprotective effects by sponging miR-873-5p}

In hypoxic and ischemic cardiomyocytes treated with Hypo-Exos, qRT-PCR revealed that inhibition of lncRNAUCA1 significantly elevated miR-873-5p in a dosedependent manner (Fig. 6a). The binding sites for IncRNA-UCA1 and miR-873-5p were examined using a luciferase reporter assay. We cloned either wild-type 3'UTR or mutant 3'UTRs of lncRNA-UCA1 in the putative miR-873-5p-binding sites into a reporter plasmid and assessed their responsiveness to miR-873-5p in 293T cells. The results showed that miR-873-5p reduced luciferase activity for IncRNA-UCA1 wild-type $3^{\prime} \mathrm{UTR}$ constructs. When one of the binding sites was mutated, luciferase activity was also reduced. These results show that lncRNA-UCA1 binds to both the binding sites (Fig. 6a).

H9c2 cells under H/SD conditions were then transfected with the miR-873-5p mimics and inhibitor (Fig. 6b). The miR-873-5p inhibitor promoted significant cardioprotective effects that were attenuated by miR- 873 mimics (Fig. 6c-g). Moreover, if hypoxic or ischemic H9c2 cardiomyocytes were transfected with a miR-873-5p mimic, 


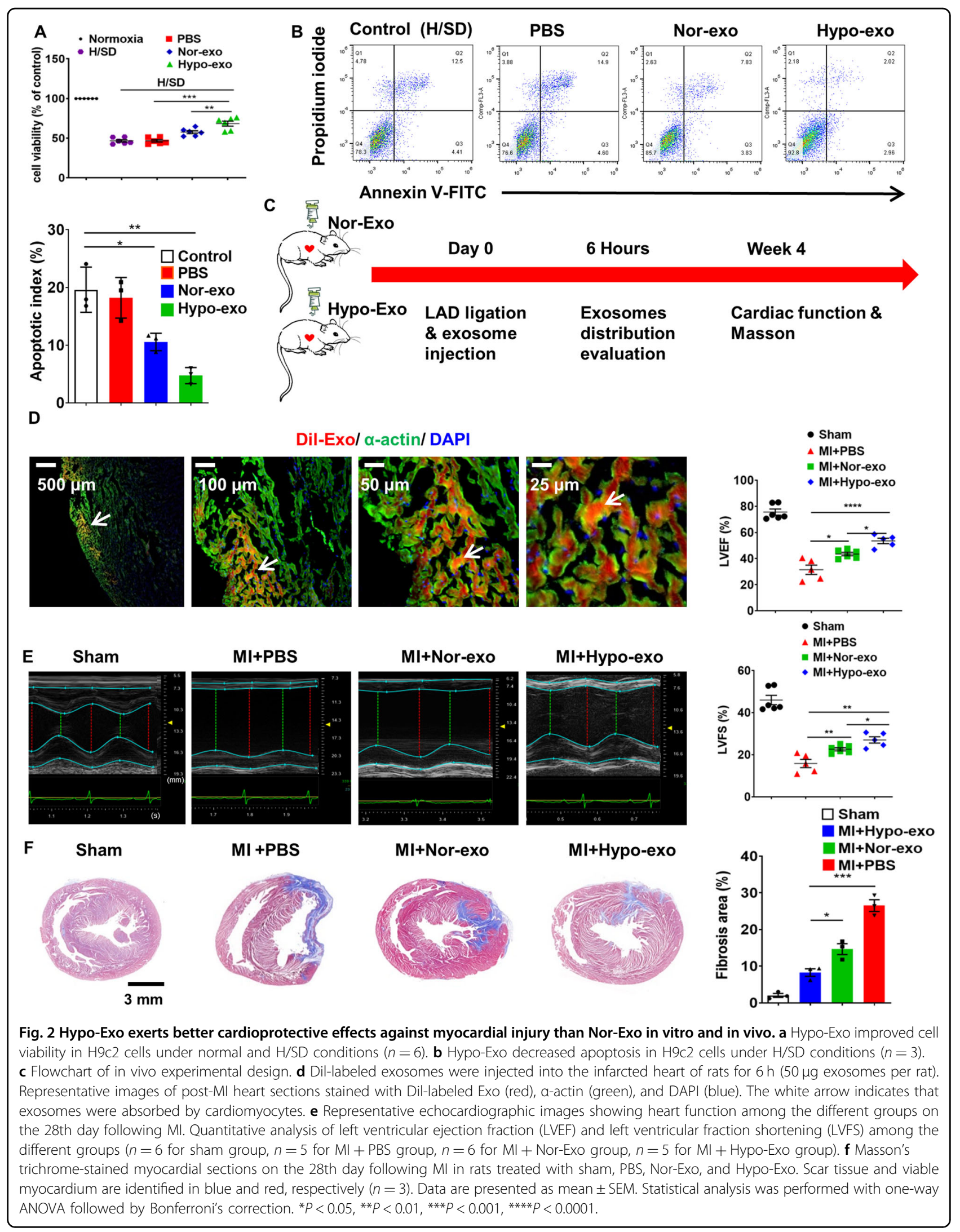




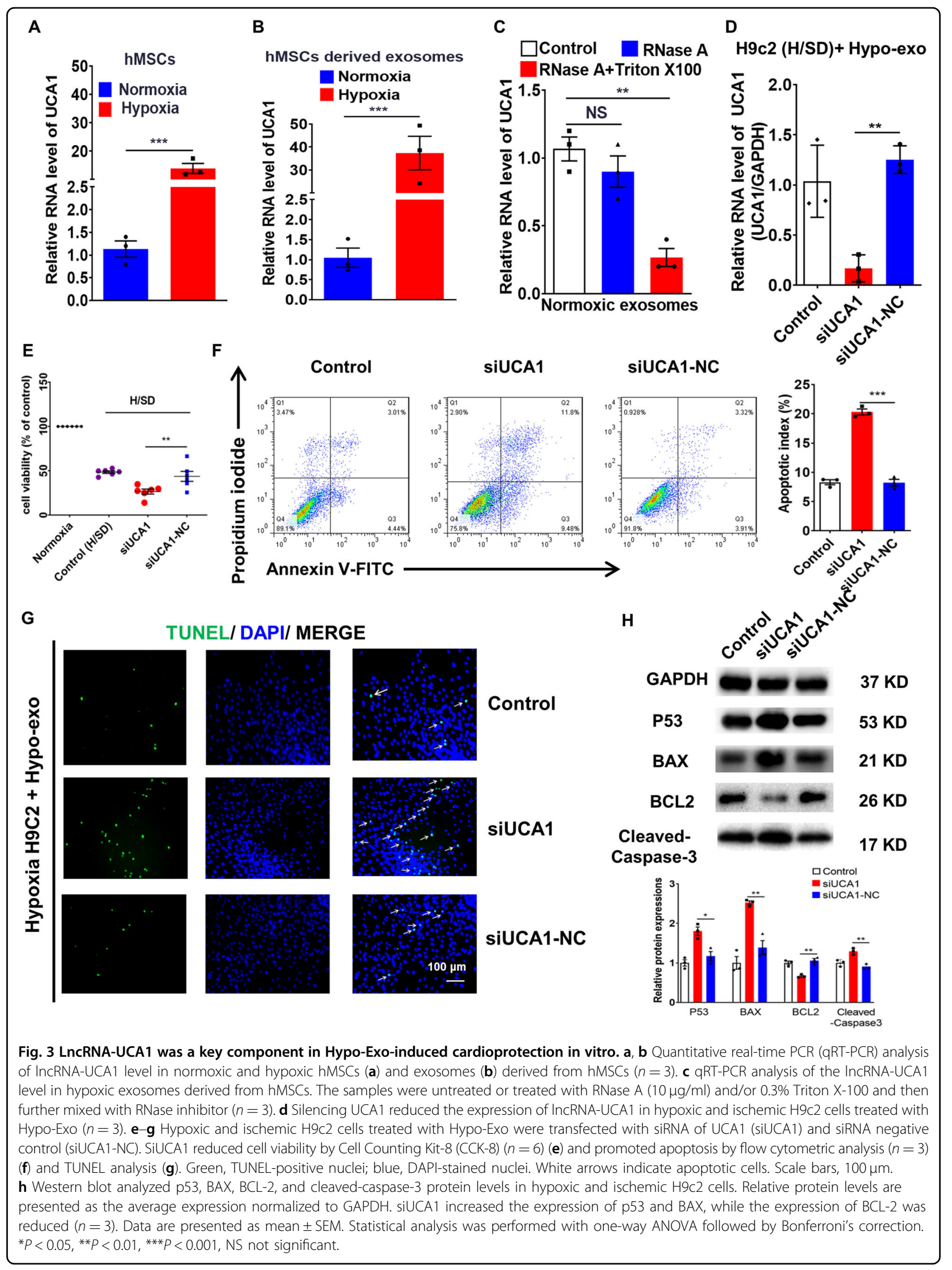




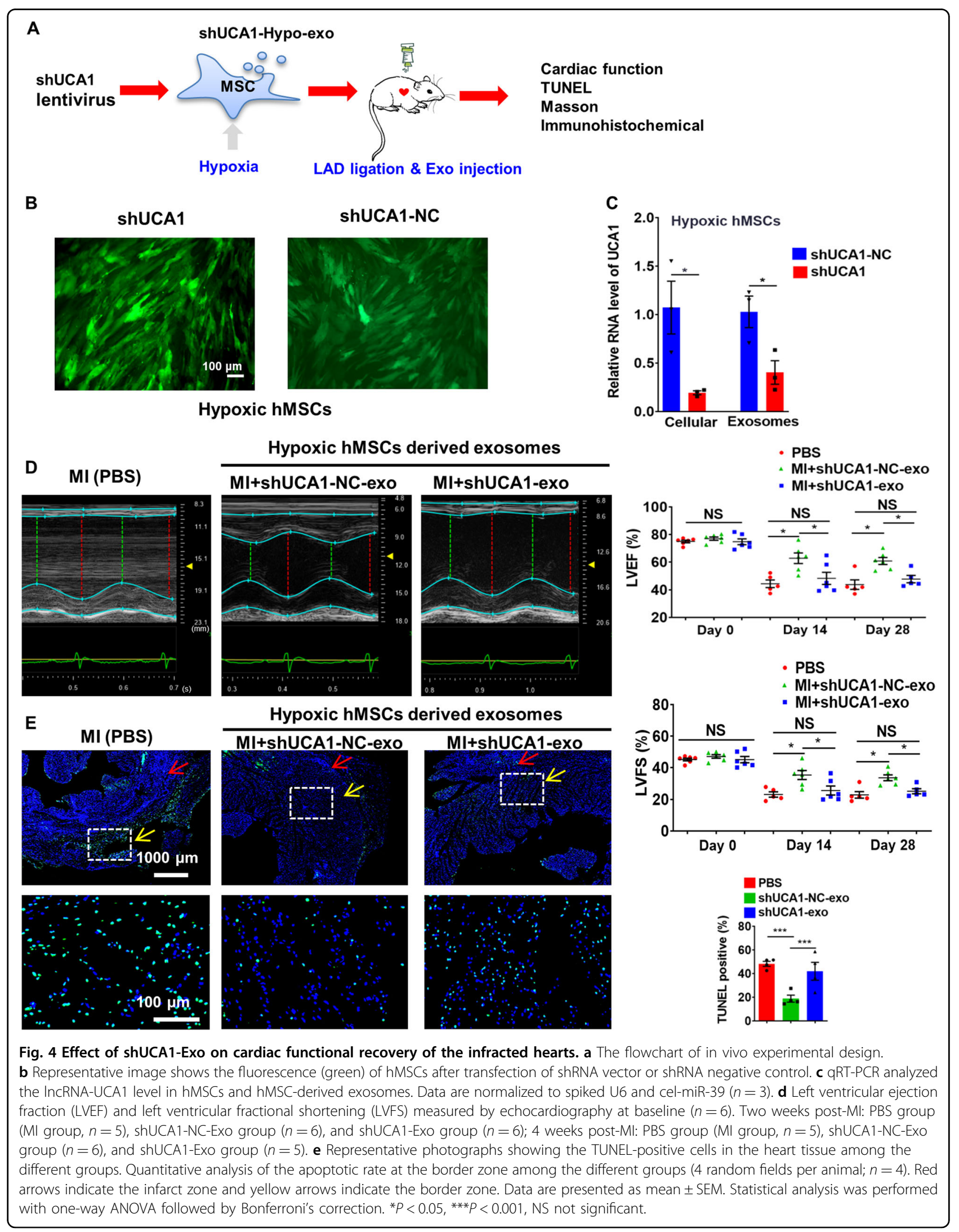




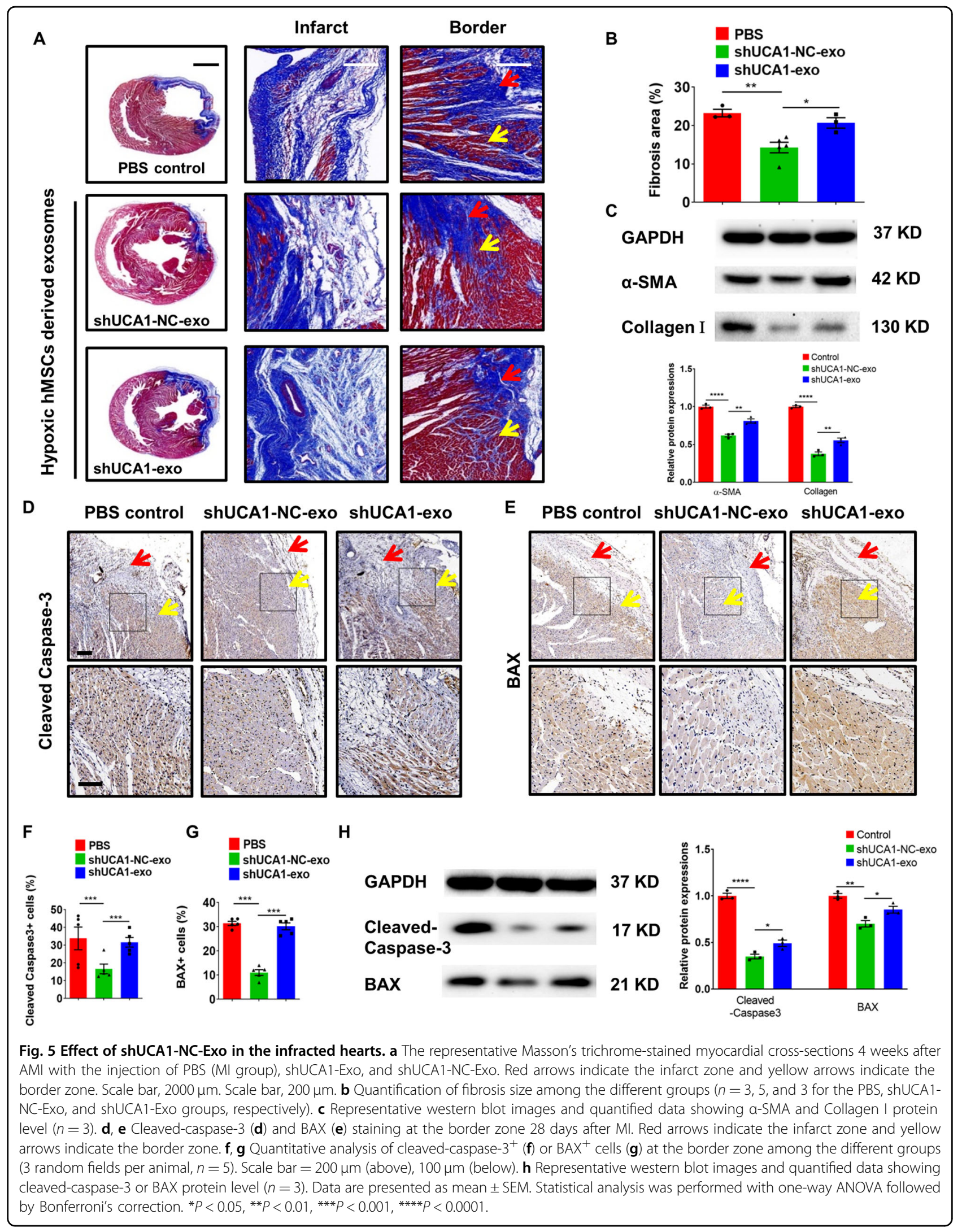




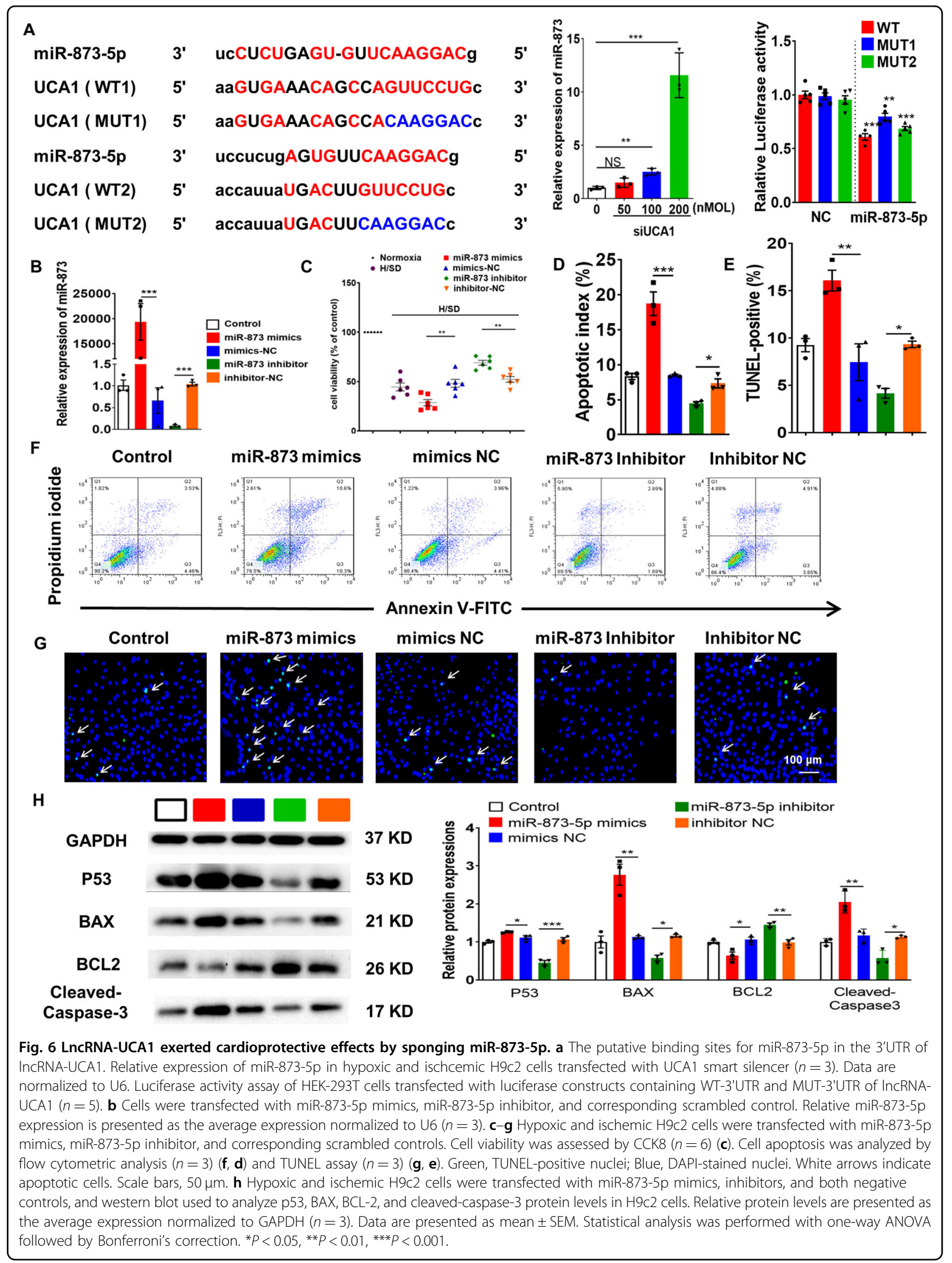


there was significant upregulation of BAX, P53, and cleaved-caspase-3 and downregulation of BCL-2 (Fig. 6f). These data demonstrate that IncRNA-UCA1 contains functional miR-873-5p-binding sites and acts as a sponge for miR-873-5p.

\section{miR-873 enhanced the phosphorylation of AMPK in cardiomyocytes by targeting XIAP}

The target genes of miR-873-5p were predicted by PITA (http://genie.weizmann.ac.il/pubs/mir07/mir07_data.html) ${ }^{19}$, miRmap (http://mirmap.ezlab.org) ${ }^{20}$, and Diana-microT (http://diana.imis.athena-innovation.gr/DianaTools/index. php? $\mathrm{r}=$ microT_CDS/index) ${ }^{21,22}$. Among the possible miR873-5p target genes that are also involved in the apoptosis pathway, we focused on XIAP (Fig. 7a). Western blot analysis showed that silencing IncRNA-UCA1 suppressed the protein level of XIAP and phosphorylation of AMPK (Fig. 7b). In addition, miR-873-5p mimics reduced the phosphorylation of AMPK and XIAP level (Fig. 7c). Transfection of miR-873 mimics was associated with a decreased protein level of XIAP (Fig. 7d). Although silencing UCA1 could increase the protein level of BAX and downregulate the protein level of BCL2 and XIAP and phosphorylation of AMPK in hypoxic H9c2 cells treated with hypoxic Exo, transfection of the miR-873 inhibitor could rescue these changes (Fig. 7e). These data suggest the essential role of the UCA1/miR-873-5p/XIAP axis in cardioprotection.

\section{Circulating exosomal IncRNA-UCA1 may be a promising novel biomarker for AMI diagnosis}

Patients with AMI $(n=26)$ and healthy donors $(n=26)$ were enrolled. The clinical characteristics of the enrolled patients are listed in Supplementary Table 4. Exo were extracted from the plasma of AMI patients and healthy donors (Fig. 8a). TEM analysis revealed that the average size of these isolated extracellular vesicles was consistent with Exo (Fig. 8b). NTA confirmed no significant differences in size distribution between the two groups (Fig. 8c). No significant differences were found in average size or concentrations between plasma-derived Exo from AMI and controls (Supplementary Table 5). Flow cytometric analysis showed that the circulating Exo were positively expressed for exosomal protein markers, such as CD81 and CD63 (Fig. 8d). Western blot analysis revealed that circulating Exo were positive for the exosome-specific markers TSG101, CD81, and CD63 (Fig. 8e). We also found that the level of exosomal lncRNA-UCA1 was markedly higher in AMI patients than in healthy volunteers (Fig. 8f). The ROC analysis indicated that circulating exosomal lncRNA-UCA1 (AUC $=0.82$ ) might be a novel diagnostic biomarker of AMI (Fig. 8g). Collectively, these data demonstrate that circulating exosomal IncRNA-UCA1 may be a promising novel biomarker for AMI diagnosis.

\section{Discussion}

There are three major findings of the current study. First, hypoxia hMSC-derived Exo transmit protective signals and endow cardioprotective effects to prevent $\mathrm{MI}$ injury. Second, we identified hMSC-derived exosomal lncRNA-UCA1 as a major cardioprotective molecule in the ischemic heart. Third, circulating exosomal lncRNAUCA1 may serve as a noninvasive biomarker for AMI diagnosis (Fig. 8h). These findings reveal a novel mechanism by which hypoxic hMSC-derived Exo exert cardioprotective effects through the exosomal lncRNAUCA1/miR873-5p/XIAP axis.

Noncoding RNAs encapsulated in Exo are particularly stable in body fluids because the RNAs are free from degradation by ribonuclease ${ }^{23}$. We confirmed that Exo provided a protective membrane for IncRNA-UCA1 against RNase degradation. Exo are released from cardiomyocytes and enter body fluids ${ }^{24}$, including plasma, serum, and urine. The level of some exosomal lncRNAs is associated with the prognosis of cardiovascular diseases and may be useful as noninvasive biomarkers ${ }^{25,26}$. The present study showed that exosomal lncRNA-UCA1 in AMI patients is much higher than in normal controls. These results provide novel evidence that circulating exosomal lncRNA-UCA1 may serve as a potential biomarker for the diagnosis of AMI.

Previous studies have shown that the plasma level of UCA1 decreases $2 \mathrm{~h}$ after AMI and reaches its lowest point after $6-12 \mathrm{~h}$. After $48-72 \mathrm{~h}$ of AMI onset, the circulating UCA1 level begins to recover and is restored to the control level. UCA1 level has been shown to be higher $72-96 \mathrm{~h}$ after AMI than in non-AMI subjects ${ }^{27}$, contrary to the results of the present study. The reason is that lncRNA was detected in plasma, whereas in the current study lncRNA was detected in plasma-derived Exo. Tian et $\mathrm{al}^{28}$ found that the level of two onco-miRNAs (miR181b-5p and miR-21-5p) was significantly higher in Exo than in plasma in lung cancer patients. Xie et al. ${ }^{29}$ performed miRNA profiling using plasma and plasmaderived Exo samples from two animal models of kidney disease and revealed that, because of the differential changes in miRNAs, measurement of exosomal miRNAs could not be replaced by measurement of miRNA in plasma or vice versa. We infer that plasma lncRNA level may differ from plasma-derived exosomal lncRNA level. $\mathrm{Yu}$ et $\mathrm{al} .{ }^{30}$ reported that plasma IncRNA UCA1 may be a good indicator for the diagnosis of chronic heart failure (CHF) and might predict poor CHF outcomes. Taken together, these studies have proposed lncRNA-UCA1 as a potential biomarker in the cardiovascular system.

Liu et al. ${ }^{31}$ reported that a decrease in UCA1 level promoted apoptosis of primary cardiomyocytes by stimulating expression of the $\mathrm{p} 27$ protein. Wang et al. ${ }^{32}$ reported that inhibition of miR-143 resulted in a 


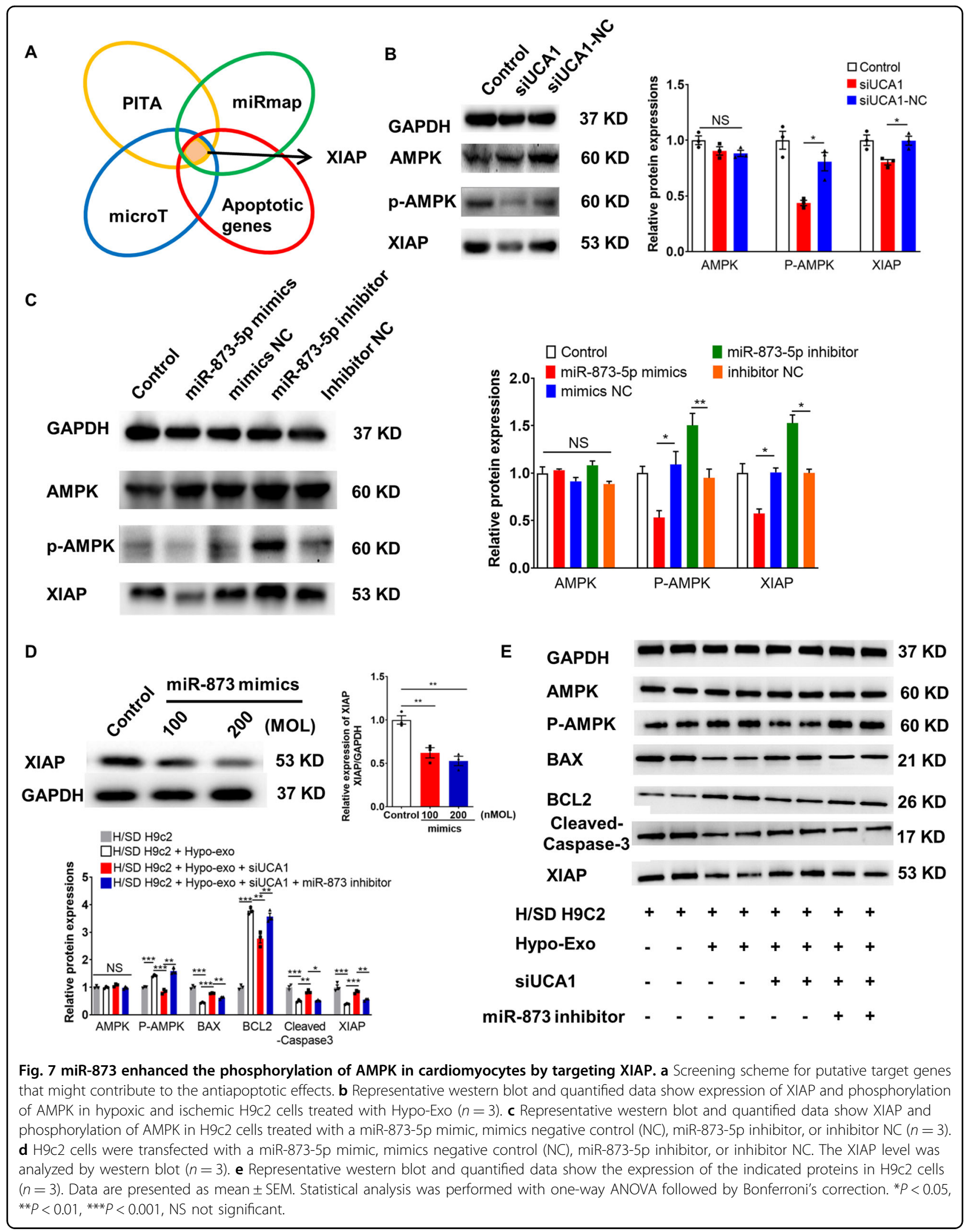




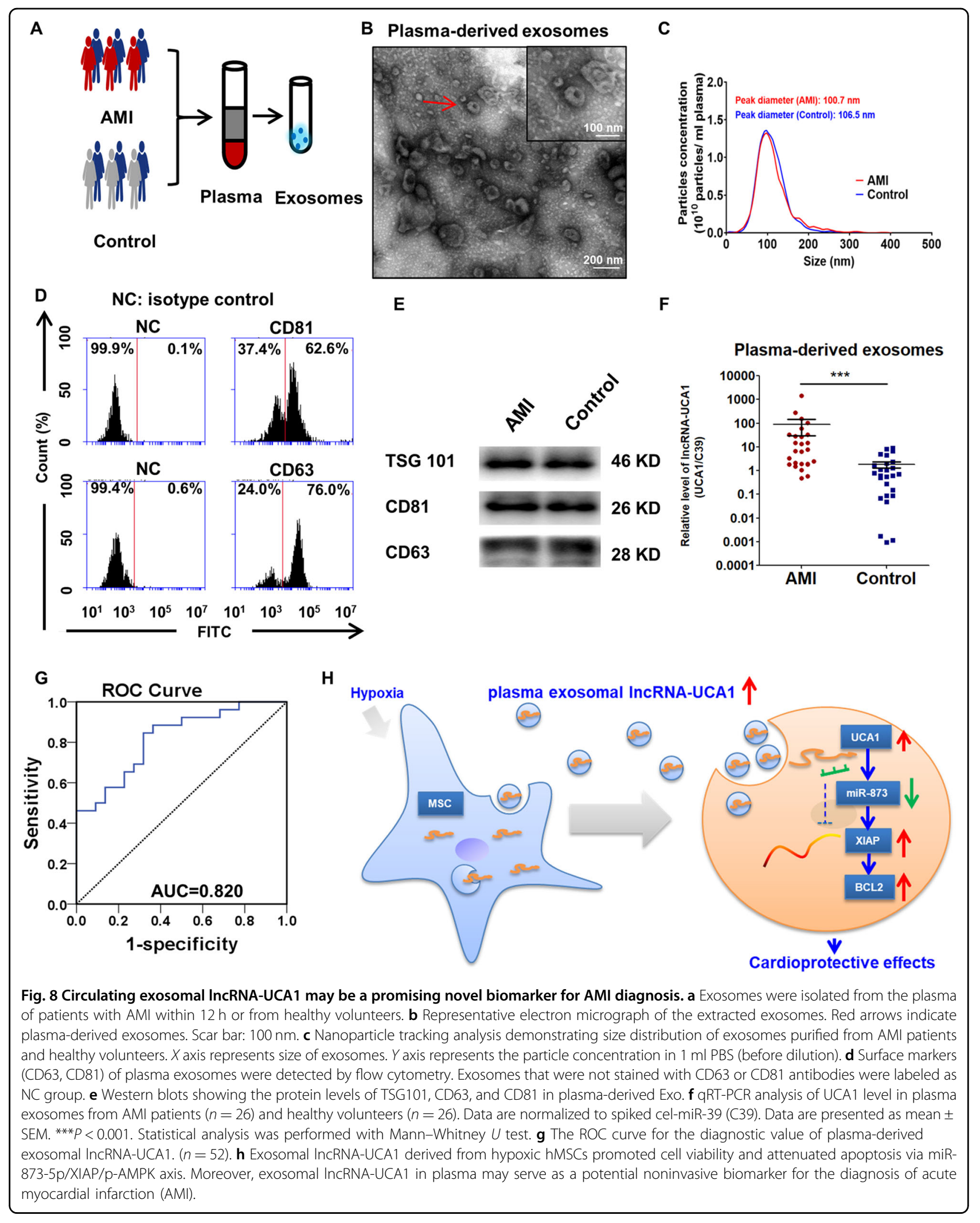


protective effect of IncRNA UCA1 on cardiomyocytes against hypoxia/reoxygenation-induced apoptosis. Our study revealed these cardiac-protective effects via exosome-derived IncRNA-UCA1 in both in vivo and in vitro experiments. These results are consistent with the observation of UCA1 expression and apoptosis following cardiac injury, suggesting that UCA1 might play an important role in the process of ischemia and hypoxia of cardiomyocytes.

Compared with liposomes and polyethylenimine, local injection of miR21-enriched extracellular vesicles from cells has demonstrated therapeutic efficacy in a mouse model of $\mathrm{MI}^{33}$. Thus cell-derived Exo may be a valuable clinical alternative for MI treatment. It has been reported that the intramyocardial delivery of Hypo-Exo in mice can reduce infarct size and enhance cardiac repair following $\mathrm{MI}$, and miR-125b has been identified as an exerkine with protective antiapoptotic effects ${ }^{13}$. Nonetheless, other noncoding RNAs may also contribute to these antiapoptotic and therapeutic effects. In our study, we speculate that hypoxic hMSCs promoted IncRNA-UCA1 transferred in Exo to mediate the phenotypes for cardiomyocytes. Our results are consistent with this hypothesis. Hypoxia promotes the secretion of IncRNA-UCA1enriched Exo from hMSCs, which play a cardioprotective role in cardiomyocytes. In addition, our study demonstrates that IncRNA-UCA1 regulates apoptosis via the miRNA-873-5p/XIAP axis. Both XIAP and BCL-2 were considered antiapoptotic genes, and it was also reported that XIAP could serve as an E3 ligase for BCL-2; thus degradation of BCL-2 by XIAP could promote apoptosis ${ }^{34}$.

The present study has some limitations. First, H9c2 cells, which are known to undergo expansive oxidative stress with further passaging ${ }^{35}$, are not a good representation of cardiomyocytes. Primary neonatal rat cardiomyocytes are a more preferable source for in vitro studies. Nonetheless, it is also reported that H9c2 cells and primary neonatal cardiomyocyte cells show similar hypertrophic responses in vitro ${ }^{36}$. Second, although intramyocardial injection is an efficient means by which to accurately deliver cardiac gene to the target area, this strategy causes damage and the injected Exo may leak at the injection site. Third, because of the lack of targeting, these Exo may also be absorbed by other cell types of the heart, leading to extra-target effects. Gene or chemical modification of extracellular vesicles may be an effective way to solve this problem. Finally, the identification of the origin of circulating Exo needs further elucidation because of the inadequate understanding of the sorting processes in determining which RNA species are packaged into Exo and the fact that exosomal RNA content does not merely parrot the RNA profile of the secreting cell.

In summary, the present study has demonstrated that hypoxia promotes hMSCs to secret IncRNA-UCA1- enriched Exo that may play a cardioprotective role in cardiac injury through the lncRNA-UCA1/miR873-5p/ XIAP axis, and exosomal lncRNA-UCA1 in human plasma may be considered a potential noninvasive biomarker for the diagnosis of AMI.

\section{Acknowledgements}

This study was supported by the National Key R\&D Program of China (2016YFE0117000), by grants from the National Natural Science Foundation of China (Grant Nos. 81901410, 81871113), by Qinghai Provincial Natural Science Fund (2017-ZJ-769), by 333 project of Jiangsu Province (BRA2017544), and by Changzhou Sci \& Tech Program (Grant No. CJ20190102).

\section{Author contributions}

L.S., W.Z., and F.Z. contributed to the design of the study. L.S., W.Z., P.Z., Q.W., B.F., Y.Z., J.Z., and Q.C. performed the experiments. L.S. and W.Z. contributed to the material support of the study. L.S., W.Z., Y.L., Q.W., and F.Z. contributed to writing the manuscript. All authors read and approved the final manuscript.

\section{Competing interests}

The authors declare no competing interests.

\section{Publisher's note}

Springer Nature remains neutral with regard to jurisdictional claims in published maps and institutional affiliations.

Supplementary Information accompanies this paper at (https://doi.org/ 10.1038/s41419-020-02783-5).

Received: 10 January 2020 Revised: 29 June 2020 Accepted: 9 July 2020 Published online: 10 August 2020

\section{References}

1. Zhou, M. et al. Mortality, morbidity, and risk factors in China and its provinces, 1990-2017: a systematic analysis for the Global Burden of Disease Study 2017. Lancet https://doi.org/10.1016/S0140-6736(19)30427-1 (2019).

2. Barnett, R. Acute myocardial infarction. Lancet 393, 2580 (2019).

3. Anderson, J. L. \& Morrow, D. A. Acute myocardial infarction. N. Engl. J. Med. 376, 2053-2064 (2017).

4. Crea, F. \& Libby, P. Acute coronary syndromes: the way forward from mechanisms to precision treatment. Circulation 136, 1155-1166 (2017).

5. Davidson, S. M. et al. Multitarget strategies to reduce myocardial ischemia/ reperfusion injury: JACC Review Topic of the Week. J. Am. Coll. Cardiol. 73 89-99 (2019).

6. Leong, D. P. et al. Reducing the global burden of cardiovascular disease, part 2. Prevention and treatment of cardiovascular disease. Circ. Res. 121, 695-710 (2017).

7. Vogel, B., Mehta, S. R. \& Mehran, R. Reperfusion strategies in acute myocardial infarction and multivessel disease. Nat. Rev. Cardiol. 14, 665-678 (2017).

8. Zhao, L. et al. Enhanced cell survival and paracrine effects of mesenchymal stem cells overexpressing hepatocyte growth factor promote cardioprotection in myocardial infarction. Exp. Cell Res. 344, 30-39 (2016).

9. Elshaer, S. L. et al. Adipose stem cells and their paracrine factors are therapeutic for early retinal complications of diabetes in the Ins2 mouse. Stem Cell Res. Ther. 9, 322 (2018).

10. Pegtel, D. M. \& Gould, S. J. Exosomes. Annu. Rev. Biochem. 88, 487-514 (2019).

11. Kelemen, E., Danis, J., Göblös, A., Bata-Csörgő, Z. \& Széll, M. Exosomal long noncoding RNAs as biomarkers in human diseases. EIFCC 30, 224-236 (2019).

12. Zhao, J. et al. Mesenchymal stromal cell-derived exosomes attenuate myocardial ischaemia-reperfusion injury through miR-182-regulated macrophage polarization. Cardiovasc. Res. 115, 1205-1216 (2019).

13. Zhu, L. P. et al. Hypoxia-elicited mesenchymal stem cell-derived exosomes facilitates cardiac repair through miR-125b-mediated prevention of cell death in myocardial infarction. Theranostics 8, 6163-6177 (2018).

14. Huang, P. et al. Atorvastatin enhances the therapeutic efficacy of mesenchymal stem cells derived exosomes in acute myocardial infarction via 
up-regulating long non-coding RNA H19. Cardiovasc. Res. https:/doi.org/ 10.1093/cvr/cvz139 (2019).

15. Gardiner, C., Ferreira, Y. J., Dragovic, R. A., Redman, C. W. \& Sargent, I. L. Extracellular vesicle sizing and enumeration by nanoparticle tracking analysis. J. Extracell. Vesicles https://doi.org/10.3402/jev.v2io.19671 (2013).

16. Xue, M. et al. Hypoxic exosomes facilitate bladder tumor growth and development through transferring long non-coding RNA-UCA1. Mol. Cancer 16, 143 (2017).

17. Madadi, S. \& Soleimani, M. Comparison of miR-16 and cel-miR-39 as reference controls for serum miRNA normalization in colorectal cancer. J. Cell. Biochem. 120, 4802-4803 (2019).

18. Kloten, V. et al. Multicenter evaluation of circulating plasma microrna extraction technologies for the development of clinically feasible reverse transcription quantitative PCR and next-generation sequencing analytical work flows. Clin. Chem. 65, 1132-1140 (2019).

19. Kertesz, M., lovino, N., Unnerstall, U., Gaul, U. \& Segal, E. The role of site accessibility in microRNA target recognition. Nat. Genet. 39, 1278-1284 (2007).

20. Vejnar, C. E., Blum, M. \& Zdobnov, E. M. miRmap web: comprehensive microRNA target prediction online. Nucleic Acids Res. 41, W165-W168 (2013).

21. Paraskevopoulou, M. D. et al. DIANA-microT web server v5.0: service integration into miRNA functional analysis workflows. Nucleic Acids Res. 41 W169-W173 (2013).

22. Reczko, M., Maragkakis, M., Alexiou, P., Grosse, I. \& Hatzigeorgiou, A. G. Functional microRNA targets in protein coding sequences. Bioinformatics $\mathbf{2 8}$ 771-776 (2012).

23. Cheng, L., Sharples, R. A., Scicluna, B. J. \& Hill, A. F. Exosomes provide a protective and enriched source of miRNA for biomarker profiling compared to intracellular and cell-free blood. J Extracell. Vesicles https://doi.org/10.3402/jev. v3.23743 (2014)

24. Cheng, M. et al. Circulating myocardial microRNAs from infarcted hearts are carried in exosomes and mobilise bone marrow progenitor cells. Nat. Commun. 10, 959 (2019).
25. Ghafarian, F. et al. The clinical impact of exosomes in cardiovascular disorders: from basic science to clinical application. J. Cell. Physiol. 234, 12226-12236 (2019).

26. Santer, L. et al. Circulating long noncoding RNA LIPCAR predicts heart failure outcomes in patients without chronic kidney disease. Hypertension 73, 820-828 (2019).

27. Yan, Y. et al. Circulating long noncoding RNA UCA1 as a novel biomarker of acute myocardial infarction. Biomed. Res. Int. 2016, 8079372 (2016).

28. Tian, F., Shen, Y., Chen, Z., Li, R. \& Ge, Q. No significant difference between plasma miRNAs and plasma-derived exosomal miRNAs from healthy people. Biomed. Res. Int. 2017, 1304816 (2017).

29. Xie, J. X. et al. MicroRNA profiling in kidney disease: plasma versus plasmaderived exosomes. Gene 627, 1-8 (2017).

30. Yu, X. et al. Plasma long noncoding RNA urothelial carcinoma associated 1 predicts poor prognosis in chronic heart failure patients. Med. Sci. Monit. 23, 2226-2231 (2017).

31. Liu, Y. et al. Long non coding RNA-UCA1 contributes to cardiomyocyte apoptosis by suppression of p27 expression. Cell. Physiol. Biochem. 35, 1986-1998 (2015).

32. Wang, Q. S., Zhou, J. \& Li, X. LncRNA UCA1 protects cardiomyocytes against hypoxia/reoxygenation induced apoptosis through inhibiting miR-143/ MDM2/p53 axis. Genomics https:/doi.org/10.1016/j.ygeno.2019.04.009 (2019).

33. Song, Y. et al. Localized injection of miRNA-21-enriched extracellular vesicles effectively restores cardiac function after myocardial infarction. Theranostics 9 2346-2360 (2019)

34. Edison, N. et al. Degradation of BCl-2 by XIAP and ARTS promotes apoptosis. Cell Rep. 21, 442-454 (2017).

35. Witek, P. et al. The effect of a number of H9C2 rat cardiomyocytes passage on repeatability of cytotoxicity study results. Cytotechnology 68, 2407-2415 (2016).

36. Watkins, S. J., Borthwick, G. M. \& Arthur, H. M. The H9C2 cell line and primary neonatal cardiomyocyte cells show similar hypertrophic responses in vitro. In Vitro Cell. Dev. Biol. Anim. 47, 125-131 (2011). 